\title{
Effect of ellipse orientation on the thermoelastic behaviour of skew laminated composite plate with elliptical cutout
}

\author{
M S R NIRANJAN KUMAR*, M M M SARCAR ${ }^{\dagger}$, V BALA KRISHNA MURTHY ${ }^{\dagger \dagger}$ and \\ K M RAO ${ }^{\#}$ \\ Production Engineering Department, V.R. Siddhartha Engineering College, Vijayawada 520 007, India \\ ${ }^{\dagger}$ College of Engineering, Andhra University, Visakhapatnam 530003 , India \\ ${ }^{\dagger}$ P.V.P. Siddhartha Institute of Technology, Vijayawada 520 007, India

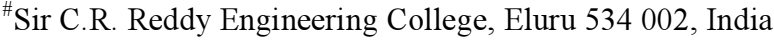

MS received 4 March 2008

\begin{abstract}
An effort is made to study the thermoelastic behaviour of a cross-ply laminated composite skew plate with elliptical cutout subjected to pressure and non-linearly varying temperature loading in the present analysis. Orientation of the elliptical cut out is varied from $0^{\circ}$ to $180^{\circ}$ with respect to horizontal at an interval of $30^{\circ}$ in the anti clockwise direction is considered for the present analysis. A three-dimensional heat conduction analysis in fibre reinforced composite laminates has been simulated by finite element method to get realistic temperature in the laminate under different thermal boundary conditions. A finite element method, which works on the basis of three-dimensional theory of elasticity, is employed to evaluate the stresses and deformations. The effect of orientation due to pressure loading on the stresses and transverse deflection is observed to be insignificant. The magnitudes of the in-plane normal stresses, $\sigma_{\mathbf{x}}$ and $\sigma_{\mathbf{y}}$, for temperature loading are greatly affected by ellipse orientation and are observed to be minimum at the ellipse orientation of $0^{\circ}$ and $90^{\circ}$, respectively. The in-plane and inter-laminar shear stresses are observed to be minimum at the ellipse orientation of $90^{\circ}$.
\end{abstract}

Keywords. FEM; skew laminate; cutout; interlaminar stresses.

\section{Introduction}

The increasing use of fibre reinforced laminates in space vehicles, aircrafts, automobiles, ships and chemical vessels have necessitated the rational analysis of structures for their mechanical response. Materials are put to serious test when they are subjected to elevated temperatures. Estimation of thermal stresses and deformations of fibre reinforced composite plates is more complex owing to material anisotropy, especially when they are subjected to combination of mechanical and thermal loading. In addition, the anisotropy and non-homogeneity and larger ratio of longitudinal to transverse modulii of these new materials demand improvement in the existing analytical tools. As a result, the analysis of laminated composite structures has attracted many research workers, and has been considerably improved to achieve realistic results. In the design of modern high-speed aircraft and missile structures, swept wing and tail surfaces are extensively employed. Moreover, some of the structural elements are provided with cutouts of different shapes to meet the functional

\footnotetext{
*Author for correspondence (m_niranjankumar@rediffmail.com)
}

requirements like (i) for the passage of various cables, (ii) for undertaking maintenance work and (iii) for fitting auxiliary equipment. Depending upon the nature of application, these structural elements are acted upon by mechanical and thermal loads of varied nature. Usually, the anisotropy in laminated composite structures causes complicated responses under different loading conditions by creating complex couplings between extensions, bending, and shear deformation modes. To capture the full mechanical behaviour, it must be described by three-dimensional elasticity theories.

In solving the three-dimensional elasticity equations of rectangular plates, quite a number of solution approaches have been proposed. Srinivas and Rao (1970) presented a set of complete analytical analyses on bending, buckling and free vibration of plates with both isotropic and orthotropic materials. Zhang et al (2002) presented a new concise procedure for obtaining the static exact solution of composite laminates with piezo-thermo-elastic layers under cylindrical bending using the basic coupled thermoelectro-elastic differential equations. Setoodeh and Karami (2004) employed a three-dimensional elasticity based layer-wise finite element method (FEM) to study the static, free vibration and buckling responses of general laminated thick composite plates. Pagano and Hatfield (1972) 
have given exact solutions for the deflections and stresses of a cross-ply laminated rectangular composites without holes using elasticity theory. Kong and Cheung (1995) proposed a displacement-based, three-dimensional finite element scheme for analysing thick laminated plates by treating the plate as a three-dimensional inhomogeneous anisotropic elastic body. Prasad and Shuart (1990) presented a closed form solution for the moment distributions around holes in symmetric laminates subjected to bending moments. Ukadgaonker and Rao (2000) gave a general solution for bending of symmetric laminates with holes. Morley (1963) developed an elementary bending theory for the small displacements of initially flat isotropic skew plates without hole. Karami et al (2003) applied differential quadrature method (DQM) for static, free vibration, and stability analysis of skewed and trapezoidal composite thin plates without hole. From the review of available literature it is observed that the static analysis of skew plates with cutouts using elasticity theory has not been studied. The behaviour of a laminate with skew edges and having various types of cutouts is different from the one without skew edges and/or cutouts. So it is necessary to analyse this kind of problem using elasticity theory based finite element method to evaluate for the most accurate behaviour of thick laminated skew plates with cutouts.

In the present analysis a rhombic laminated composite skew plate is considered by varying the orientation of the elliptical cut out (figure 1).

\section{Problem statement}

The research problem deals with the thermoelastic analysis of thick skew laminated plate with elliptical cutout by elasticity theory based on finite element method.

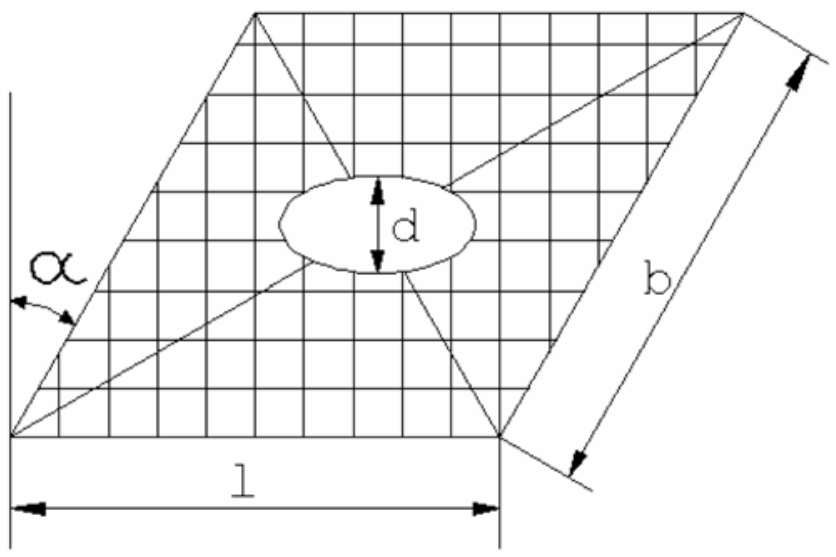

Figure 1. Skew laminated composite plate with elliptical cutout.

\section{Problem modeling}

\subsection{Geometric modeling}

Figure 1 shows the in-plane dimensions of the laminate considered for the present analysis. The dimensions for ' $l$ ' and ' $b$ ' are taken as $20 \mathrm{~mm}$. $d$ is the length of the minor axis of the ellipse. Major axis of the ellipse is taken as twice the length of the minor axis.

The value of $d$ is determined from the ratio of $d / l$ which is taken to be $0 \cdot 3$, and the skew angle, $\alpha$, is taken to be $30^{\circ}$, the thickness of the plate is fixed from the length to thickness ratio, $l / h(s=10)$. The individual layers are arranged so that the total thickness of the layers oriented in $x$-direction $\left(\theta=0^{\circ}\right)$ is equal to the total thickness of the layers oriented in $y$-direction $\left(\theta=90^{\circ}\right)$.

\subsection{Finite element modeling}

The finite element mesh is generated using a three-dimensional brick elements 'SOLID 90' and 'SOLID 95' of ANSYS (ANSYS Reference Manual 2006). The 20 node thermal element is applicable to a steady state or transient thermal analysis. The elements consist of 20 nodes and temperature degree of freedom for 'SOLID 90' and $X, Y$ and $Z$ directional displacement for 'SOLID 95'. 'SOLID 90' and 'SOLID 95' have compatibility to transfer the temperatures from thermal analysis to structural analysis.

\section{Boundary conditions}

\subsection{Thermal}

A temperature of $100^{\circ} \mathrm{C}$ on the top face and $25^{\circ} \mathrm{C}$ on the bottom and side faces is applied. The surface of the hole is subjected to convection with film coefficient, $h=5$ and bulk temperature, $25^{\circ} \mathrm{C}$.

\subsection{Structural}

All the edges of the skew plate are clamped i.e. all the three degrees of freedom (Displacements in global $x$-, $y$ and $z$-directions) of the nodes attached to the side faces of the plate are constrained.

\section{Loading}

(i) The output from the thermal analysis is applied as thermal loading.

(ii) A transverse pressure of $1 \mathrm{MPa}$ is applied on the top surface of the plate in addition to the change in temperature in case of combined loading. 
Table 1. Comparison of present work with exact elasticity theory.

\begin{tabular}{|c|c|c|c|c|c|c|c|c|c|c|}
\hline$\frac{S=l / h}{10}$ & \multicolumn{2}{|c|}{$\begin{array}{c}\text { Normalized } \\
\sigma_{x}(a / 2, a / 2, \pm 1 / 2)\end{array}$} & \multicolumn{2}{|c|}{$\begin{array}{c}\text { Normalized } \\
\sigma_{y}(a / 2, a / 2, \pm 1 / 3)\end{array}$} & \multicolumn{2}{|c|}{$\begin{array}{c}\text { Normalized } \\
\sigma_{y z}(0, a / 2,0)\end{array}$} & \multicolumn{2}{|c|}{$\begin{array}{c}\text { Normalized } \\
\sigma_{z x}(a / 2,0,0)\end{array}$} & \multicolumn{2}{|c|}{$\begin{array}{c}\text { Normalized } \\
w(a / 2, a / 2,0)\end{array}$} \\
\hline 10 & $\begin{array}{l}\text { EL } \\
\text { FE }\end{array}$ & $\begin{array}{r}0.545 \\
-0.545 \\
0.537 \\
-0.536\end{array}$ & EL & $\begin{array}{r}0.430 \\
-0.432 \\
0.431 \\
-0.431\end{array}$ & EL & $\begin{array}{l}0.223 \\
0 \cdot 209\end{array}$ & EL & $\begin{array}{l}0.258 \\
0 \cdot 212\end{array}$ & $\begin{array}{l}\mathrm{EL} \\
\mathrm{FE}\end{array}$ & $\begin{array}{l}0.677 \\
0.692\end{array}$ \\
\hline 20 & EL & $\begin{array}{r}0.539 \\
-0.539 \\
0.534 \\
-0.535\end{array}$ & EL & $\begin{array}{c}0 \cdot 380 \\
-0 \cdot 380 \\
0 \cdot 377 \\
-0.378\end{array}$ & EL & $0 \cdot 212$ & EL & $\begin{array}{l}0 \cdot 268 \\
0 \cdot 271\end{array}$ & $\begin{array}{l}\text { EL } \\
\text { FE }\end{array}$ & $\begin{array}{l}0.4938 \\
0.4838\end{array}$ \\
\hline
\end{tabular}

\section{Material properties (graphite-epoxy)}

$K_{\mathrm{L}}=36 \cdot 42 \mathrm{~W} / \mathrm{m} \mathrm{K}$,

$E_{1}=172.72 \mathrm{GPa}$,

$K_{\mathrm{T}}=0.96 \mathrm{~W} / \mathrm{m} \mathrm{K}$

$G_{12}=G_{13}=3.45 \mathrm{GPa}$,

$E_{2}=E_{3}=6.909 \mathrm{GPa}$,

$G_{23}=1.38 \mathrm{GPa}$,

$v_{12}=v_{13}=v_{23}=0.25$ and

$\alpha_{1}=0.57 \times 10^{-6} /{ }^{\circ} \mathrm{C}$,

\section{Validity of present analysis}

To validate the finite element results, a square plate with simply supported edges and subjected to a sinusoidal load of $p=p_{0} \sin (\pi x / a) \sin (\pi y / b)$, where $a$ and $b$ are the length and width of the plate is modeled with SOLID 95 element. The results obtained from this model are compared with the exact elasticity solution for various lengths to thickness ratios of the plate (table 1). It is observed that the finite element results are in close agreement with the exact elasticity solution.

Table 1 presents the results of a square laminate $\left({ }^{\prime} a{ }^{\prime}={ }^{\prime} b\right.$ '). The location for maximum $\sigma_{x}$ i.e. $(a / 2, a / 2$, $\pm 1 / 2)$ is at the centre and at top and bottom faces of the laminate. Maximum value of $\sigma_{y}$ is observed at the centre and at the interface of outer and its adjacent layers $(a / 2, a / 2, \pm 1 / 3)$ of the laminate. The value of the shear stress, $\tau_{y z}$, is taken at the mid point of one of the vertical sides and at the neutral surface of the laminate $(0$, $a / 2,0)$.

The value of the shear stress, $\tau_{z x}$, is taken at the mid point of one of the horizontal sides and at the neutral surface of the laminate $(a / 2,0,0)$ and the transverse deflection is obtained at geometric centre of the laminate $(a / 2, a / 2,0)$.

In the present work the transverse deflection and stresses (including the inter-laminar stresses at the free edge of the elliptical cutout) of a clamped skew laminated plate with elliptical cutout at the centre of the plate and subjected to pressure, thermal and combined thermal and pressure loading is evaluated by varying the orientation of the elliptical cutout.

\section{Results and discussion}

Numerical results are obtained for the three different load cases as mentioned above. Variation of the stresses and deflection with respect to the orientations of the elliptical cut out is shown in figures $2-8$. The following observations are made.

For pressure loading there is no significant change in in-plane normal stress, $\sigma_{x}$, with respect to orientation of elliptical cutout. The variation of $\sigma_{x}$ with respect to elliptical cutout orientation in $x-y$ coordinate system is the resultant effect of the following two factors.

(i) When the ellipse angle is $0^{\circ}$ (horizontal), the in plane normal stress, $\sigma_{x}$, should be minimum as the discontinuity length normal to $x$-axis which is equal to the minor axis of the ellipse which is minimum. As the orientation of the ellipse changes from $0^{\circ}$ to $90^{\circ}$ the discontinuity length normal to $x$-axis increases which increases the magnitude of $\sigma_{x}$ up to $90^{\circ}$ and later decreases at the same rate from $90^{\circ}$ to $180^{\circ}$

(ii) When the orientation of the ellipse is at $0^{\circ}$ (horizontal), since the major axis of the ellipse is parallel to the major span of the plate, the stress should be maximum at this orientation and decreases with increase in ellipse orientation up to $90^{\circ}$ and later increases from $90^{\circ}$ to $180^{\circ}$.

The second factor may be balancing the effect of first factor resulting in maintaining the same stress at all ellipse orientations.

In case of temperature loading this stress increases with increase in elliptical cutout orientation from $0^{\circ}$ to $90^{\circ}$ and then decreases from $90^{\circ}$ to $180^{\circ}$. The variation of $\sigma_{x}$ with respect to elliptical cutout orientation in $x-y$ coordinate system is due to the following reasons.

(i) Thermal stress in the laminate is due to the restriction of the laminate with constraints. Whenever the major axis of the ellipse moves away from the resultant compressive force in $x$-direction the normal stress $\left(\sigma_{x}\right)$ will increase and it is maximum when the major axis is perpendicular to the direction of compressive force i.e. when ellipse orientation is $90^{\circ}$. When the ellipse orientation is at $0^{\circ}$ this stress is minimum and increases as the ellipse orien- 
tation changes from $0^{\circ}$ to $90^{\circ}$ and decreases at the same rate from $90^{\circ}$ to $180^{\circ}$.

(ii) The thermal stress is due to the mismatch of coefficient of thermal expansion at the interfaces.

(iii) Due to the uneven expansions because of the nonlinear distribution of the temperature.

In case of combined loading this stress increases with increase in elliptical cutout orientation from $0^{\circ}$ to $90^{\circ}$ and then decreases from $90^{\circ}$ to $180^{\circ}$. The variation of $\sigma_{x}$ with respect to elliptical cutout orientation due to combined loading is the resultant of this stress in pressure loading and temperature loading (figure 2).

There is no significant change in in-plane normal stress, $\sigma_{y}$, with respect to orientation of elliptical cutout for pressure loading. For temperature loading this stress

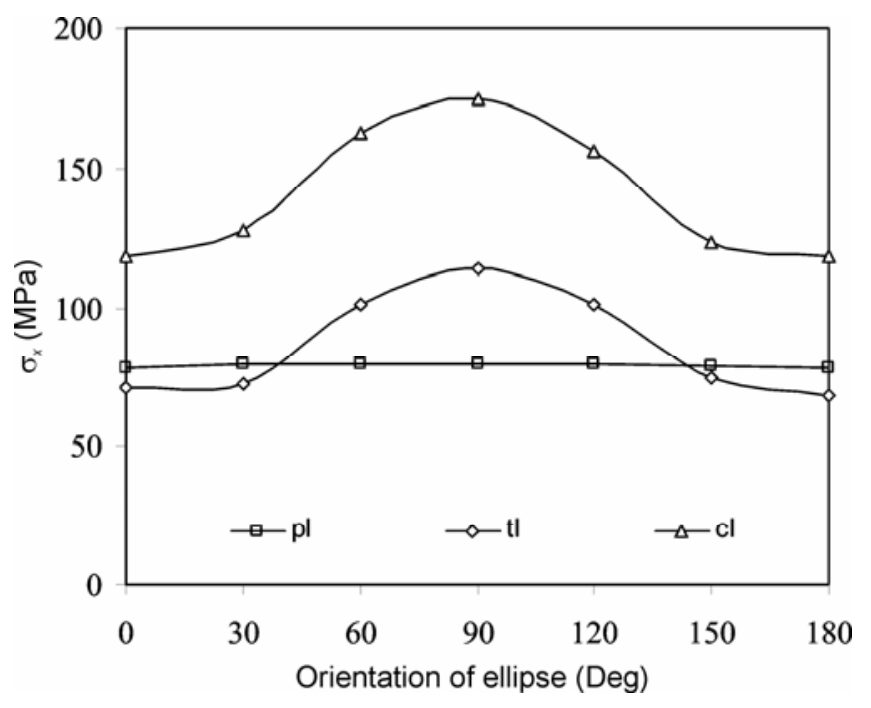

Figure 2. Variation of $\sigma_{x}$ with respect to ellipse orientation.

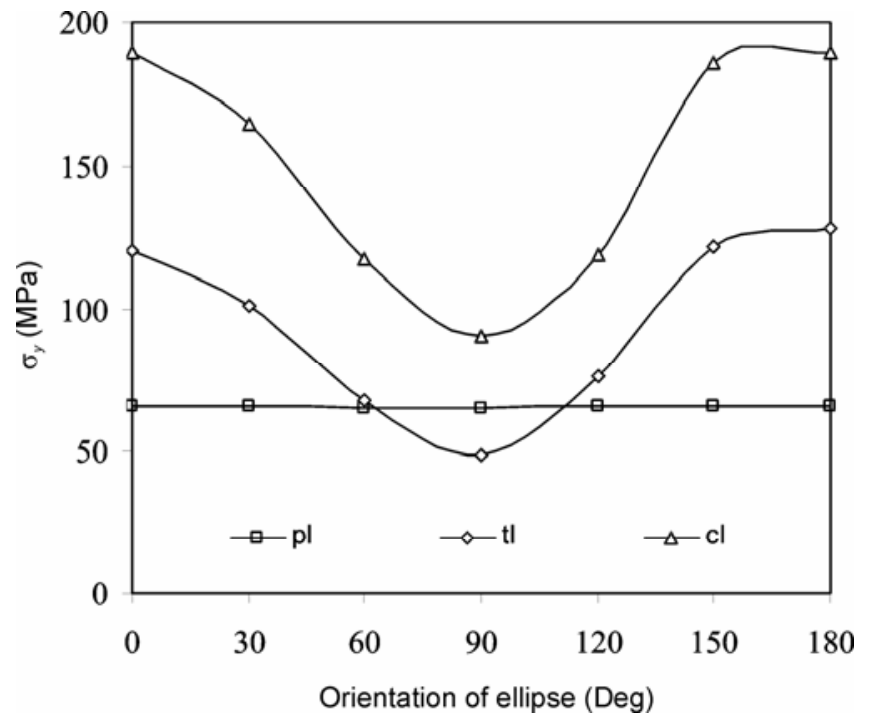

Figure 3. Variation of $\sigma_{y}$ with respect to ellipse orientation. decreases with increase in elliptical cutout orientation from $0^{\circ}$ to $90^{\circ}$ and then increases from $90^{\circ}$ to $180^{\circ}$. For combined loading this stress decreases with increase in elliptical cutout orientation from $0^{\circ}$ to $90^{\circ}$ and then increases from $90^{\circ}$ to $180^{\circ}$. The reasons for variation of this stress are similar to that explained for $\sigma_{x}$ with the stresses considered in the direction of $y$-axis (figure 3 ).

There is no significant variation of the in plane shear stress, $\tau_{x y}$, observed with respect to ellipse orientation for pressure loading. For temperature and combined loading this stress increases with increase in ellipse orientation up to $60^{\circ}$ and then decreases up to $90^{\circ}$ and again increases up to $150^{\circ}$ and later decreases. Change in $\tau_{x y}$ is due to the net effect of change in reaction moment and change in the resisting material with the change in orientation of the ellipse. $\tau_{x y}$ decreases when the resultant moment in $x y$ -

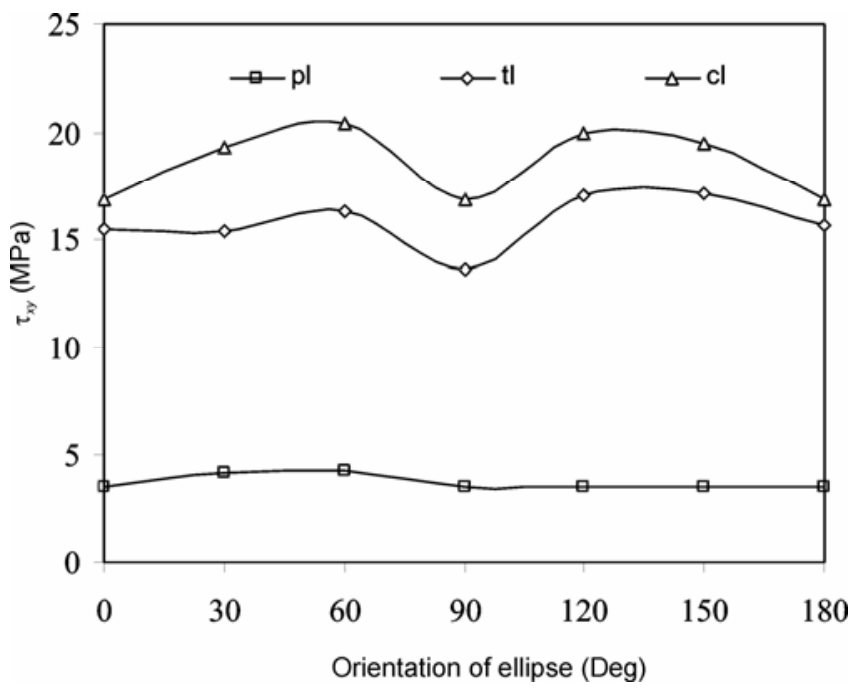

Figure 4. Variation of $\tau_{x y}$ with respect to ellipse orientation.

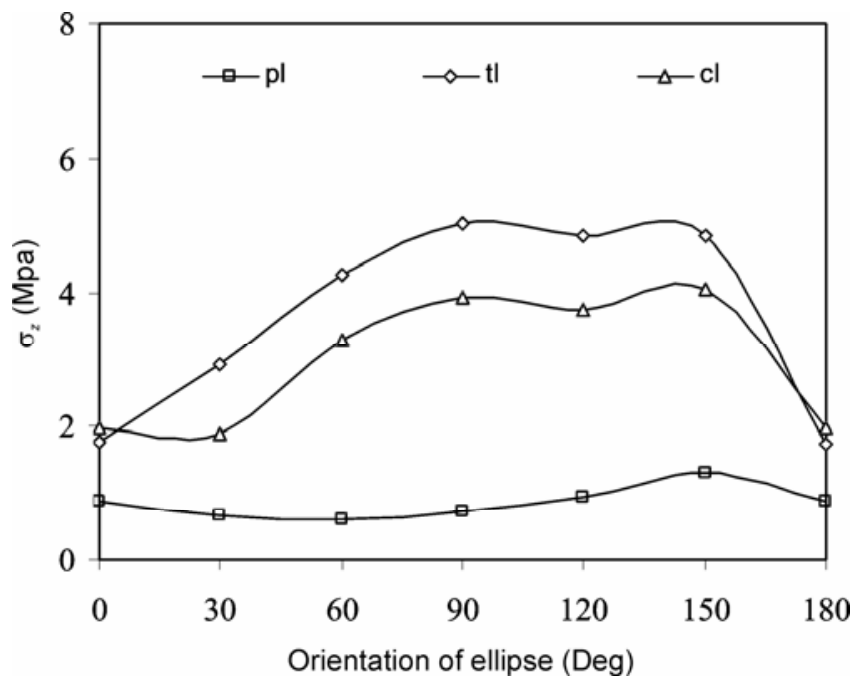

Figure 5. Variation of $\sigma_{z}$ with respect to ellipse orientation. 
plane decreases and increases when the resultant moment increases (figure 4).

There is no significant variation of the inter-laminar normal stress, $\sigma_{z}$, with respect to ellipse orientation for pressure loading. For temperature and combined loading this stress increases with increase in ellipse orientation up to $90^{\circ}$ and later decreases. The forces causing the interlaminar stresses form in couples to balance the forces for equilibrium (figure 5).

There is no significant variation of the inter-laminar shear stress, $\tau_{y z}$, with respect to ellipse orientation for pressure loading. For temperature and combined loading this stress increases with increase in ellipse orientation up to $60^{\circ}$ and later decreases up to $90^{\circ}$ and again increases up to $120^{\circ}$ and later decreases (figure 6).

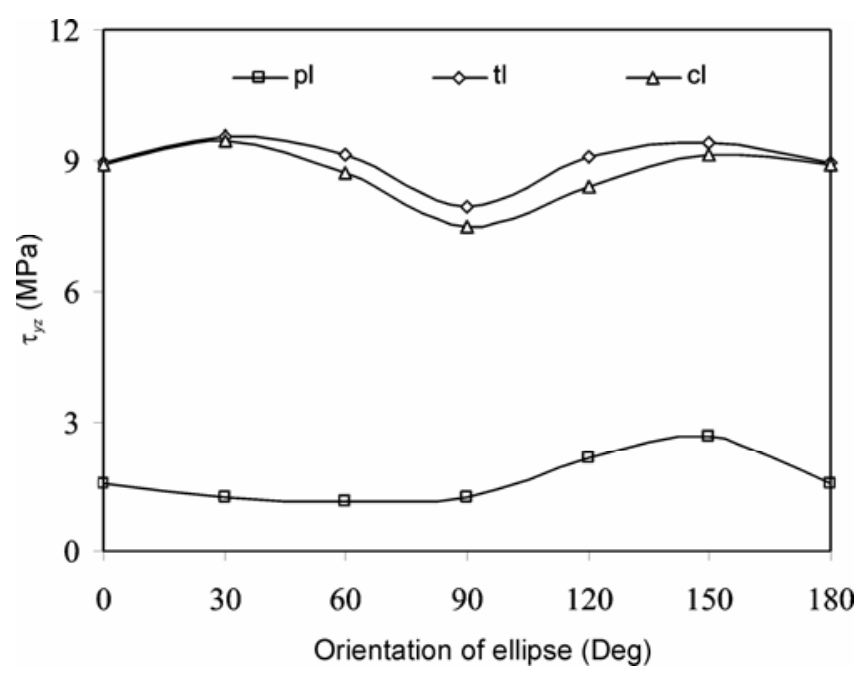

Figure 6. Variation of $\tau_{y z}$ with respect to ellipse orientation.

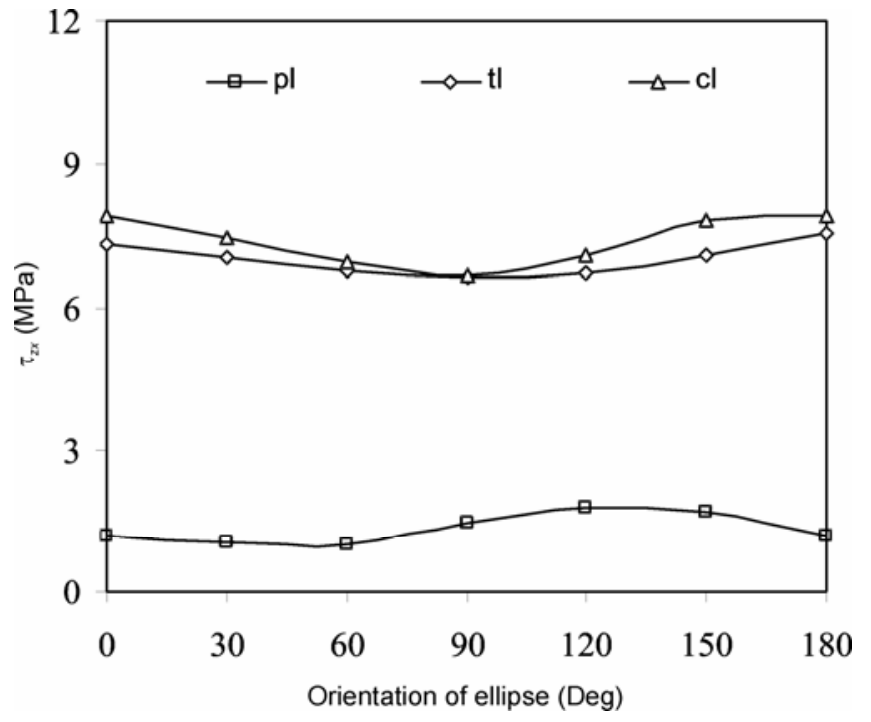

Figure 7. Variation of $\tau_{z x}$ with respect to ellipse orientation.
There is no significant variation of the inter-laminar shear stress, $\tau_{z x}$, with respect to ellipse orientation for pressure loading. For temperature and combined loading this stress increases with increase in ellipse orientation up to $90^{\circ}$ and later decreases up to $120^{\circ}$ and again increases up to $150^{\circ}$ and later decreases. However, the magnitude of these stresses is less when compared to that of in-plane stress (figure 7).

There is no significant variation of transverse deflection ' $w$ ' with respect to ellipse orientation (figure 8).

\section{Conclusions}

The results obtained for pressure, thermal and combined pressure and non-linearly varying temperature loading are analysed for the variation of elliptical cutout orientation. The effect of orientation due to pressure loading on the stresses and transverse deflection is observed to be insignificant. For temperature loading the in-plane normal stress, $\sigma_{x}$ and inter-laminar normal stress, $\sigma_{z}$ are observed to be minimum at the ellipse orientation of $0^{\circ}$ (major axis parallel to the horizontal sides of the plate). The in-plane normal stress, $\sigma_{y}$, is observed to be minimum at the ellipse orientation of $90^{\circ}$ (major axis perpendicular to the horizontal sides of the plate). Since the major stresses, $\sigma_{x}$ and $\sigma_{y}$, are moderate in between ellipse orientation of $30^{\circ}$ and $60^{\circ}$ the intermittent ellipse orientation angle of $45^{\circ}$ may be the best orientation. The in-plane and interlaminar shear stresses are observed to be minimum at the ellipse orientation of $90^{\circ}$. If the design requirement is based on in plane strength, ellipse orientation angle of $45^{\circ}$ may be preferred. If it is based on shear strength, ellipse orientation angle of $90^{\circ}$ can be preferred. Combined loading effect is the resultant of pressure and temperature loading. However, the magnitude of shear stresses is observed to be less as compared to that of normal

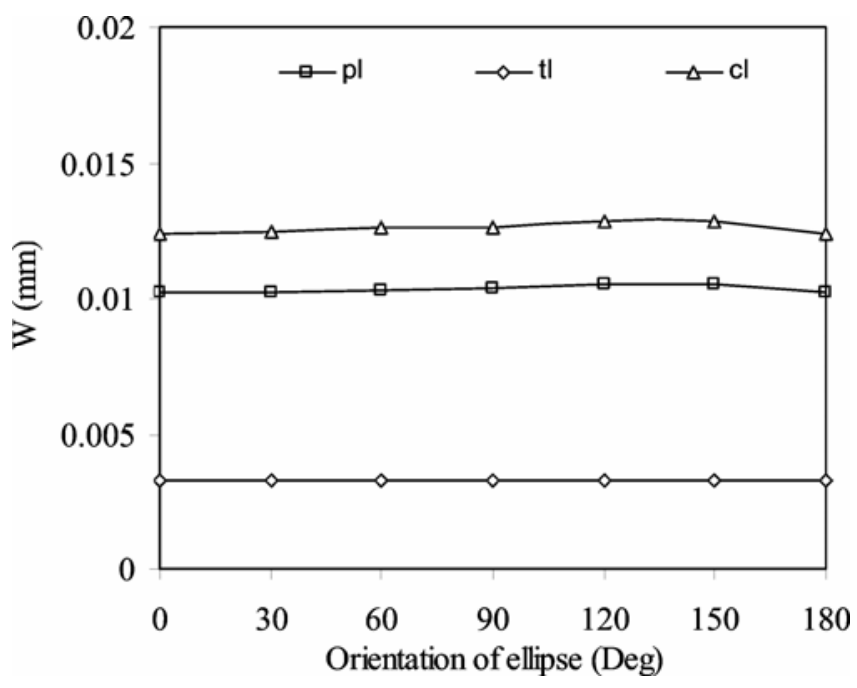

Figure 8. Variation of ' $w$ ' with respect to ellipse orientation. 
stresses. Since there is no variation of the stresses with ellipse orientation for pressure loading, the variation of stresses for combined loading is similar to that of temperature loading. The solutions of skew structures considered in the present analysis will be useful for the construction of safe and efficient structures like skew bridges and swept wings of aircraft structures.

\section{Nomenclature}

$\begin{array}{ll}\text { Symbol } & \text { Meaning } \\ \text { pl } & \text { Pressure loading } \\ \text { tl } & \text { Thermal loading } \\ \text { cl } & \text { Combined loading length of the plate }(l) / \\ s & \text { thickness of the plate }(h) \\ \text { Normalized } \sigma & \sigma / p_{0} s^{2} \\ \text { Normalized } \tau & \tau / p_{0} S \\ \text { Normalized } w & 100 E_{2} w / p_{0} h s^{4} \\ p_{0} & \text { the maximum intensity of sinusoidal load } \\ a & \text { length and width of the square plate }\end{array}$

Normalized $z$

$2 z / h, z$ coordinate measured from middle plane of the plate and $h=$ total thickness of the plate

\section{References}

Karami G, Shahpari S A and Malekzadeh P 2003 Comp. Struct. 59393

Kong J and Cheung Y K 1995 Computers and Structures 57 1051

Morley L S D 1963 Int. series of monographs on aeronautics and astronautics (New York: Pergamon Press)

Pagano N J and Hatfield S J 1972 AIAA J. 10931

Prasad C B and Shuart M J 1990 AIAA J. 28877

Setoodeh A R and Karami G 2004 Eng. Struct. 26211

Srinivas S and Rao A K 1970 Int. J. Solids \& Struct. 61463

Srinivas S, Rao C V and Rao A K 1970 J. Sound Vibrations 12 87

Ukadgaonker V G and Rao D K N 2000 Comp. Struct. 49 41

Zhang C, Di S and Zhang N 2002 Comp. Struct. 56131 